\title{
Different methods of cooking starchy-carbohydrate food and its impact on postprandial lipid metabolism
}

\author{
R. Oraee, A. Alzaabi, M.D. Robertson and B.A. Fielding \\ Department of Nutritional Sciences, Faculty of Health and Medical Sciences, University of Surrey, \\ Guildford, GU2 7WG, UK
}

There is growing evidence that both elevated plasma postprandial triacylglycerol (TAG) and glucose concentrations are associated with the development of type 2 diabetes and cardiovascular disease ${ }^{(1,2)}$. Therefore nutritional strategies to improve health through reduction of postprandial TAG and glucose are important. It is well known that cooking and cooling food forms retrograded starch (RS3) and leads to a reduction in postprandial plasma glucose concentrations ${ }^{(3)}$. Furthermore it has been shown that chilling and reheating starchy food in the presence of lipid forms a new type of resistant starch (RS5; an amylose-lipid complex). However, no studies have investigated postprandial TAG lipaemia with respect to mixed meals containing naturally forming resistant starch ${ }^{(4)}$.

The present study aims to investigate the effect cooking and reheating a starchy-carbohydrate meal containing lipid on postprandial lipid metabolism. The study hypothesised that RS5 formation after chilling and reheating the meal would significantly decrease the postprandial lipid response.

A randomised, cross-over pilot study was performed in five healthy individuals' aged $24.6 \mathrm{y} \pm 3.64$ and with BMI of $23.9 \mathrm{~kg} / \mathrm{m}^{2} \pm 2 \cdot 09$. Participants were randomly allocated to consume one of four different test meals on each of four study visits. Each meal contained $300 \mathrm{~g}$ of mashed potatoes ( $50 \mathrm{~g}$ carbohydrate), and either $15 \mathrm{~g}$ or $30 \mathrm{~g}$ butter fat. For each fat content, the meals were either served freshly cooked or cooked the previous evening and reheated on the day of the study in a microwave (to give 4 combinations). Blood samples were taken at baseline (fasting) and for the 6-h after meal consumption to determine the plasma glucose, TAG and NEFA concentrations.

The mean iAUC for the postprandial TAG response was significantly lower (Meal C; $88.479 \mathrm{mmol} / 1.360 \mathrm{~min}, \pm 12.7)(\mathrm{Meal} \mathrm{D}$; $51.642 \mathrm{mmol} / 1.360 \mathrm{~min}, \pm 13 \cdot 2) . P=0.043$ in the individuals who consumed the chilled and reheated mashed potato with $30 \mathrm{~g}$ of fat compared to those eating the same meal freshly cooked. There was no statistically significant difference in the lower-fat ( $15 \mathrm{~g}$ ) meals. However, the data showed a trend towards a decrease in the TAG response. The mean AUC for NEFA response in the plasma was also lower in the chilled and reheated meals with both fat doses. However, this was not statistically significant. There was no statistically significant difference in the glucose response between different meals.

For the first time, we have shown that reheating a starchy carbohydrate meal in the presence of fat significantly lowers the postprandial TAG response. This simple cooking procedure could have implications for cooking at home as well as reheating ready meals. Developing this pilot study further, including in vivo stable isotope studies and in vitro digestibility experiments will help to determine the mechanisms behind our findings.

1. Ceriello A (2004) Postprandial Hyperglycemia and Diabetes Complications: Is It Time to Treat? Diabetes 54(1): 1-7.

2. Parks E (2001) Recent findings in the study of postprandial lipemia. Current Atherosclerosis Reports 3(6): 462-470.

3. Robertson M (2012) Dietary-resistant starch and glucose metabolism. Current Opinion in Clinical Nutrition and Metabolic Care 15(4): 362-367.

4. Fuentes-Zaragoza E, Riquelme-Navarrete M, Sánchez-Zapata E, Pérez-Álvarez J. (2010) Resistant starch as functional ingredient: A review. Food Research International 43(4): 931-942. 